\title{
Sensor Placement for Structural Damage Detection Considering Measurement Uncertainties
}

\author{
Xiao-Qing Zhou', Yong $\mathrm{Xia}^{2}{ }^{2 *}$ and Hong $\mathrm{Hao}^{3}$ \\ ${ }^{1}$ College of Civil Engineering, Shenzhen University, Shenzhen, China \\ ${ }^{2}$ Department of Civil and Environmental Engineering, The Hong Kong Polytechnic University, Hong Kong, China \\ ${ }^{3}$ School of Civil and Resource Engineering, The University of Western Australia, Australia
}

(Received: 8 June 2012; Received revised form: 26 January 2013; Accepted: 5 February 2013)

\begin{abstract}
Structural damage detection methods using vibration measurements have been developed for decades. Measurement selections may affect damage detection results, because inevitable uncertainties are involved in vibration testing. A new sensor placement index is defined as the ratio of two parameters, namely, the contribution of measurement points to a Fisher information matrix, and the damage sensitivity to the measurement noise. A large value of the contribution vector represents that the corresponding measurement points are sensitive to the damage and measurements at these points are more prominent for structural damage identification, whereas a small noise sensitivity value indicates measurement points that are less influenced by noises. Consequently, the points with large index values are chosen as the measurement subset. The effectiveness of the proposed technique is verified using a laboratorytested steel frame. The damage detection using different measurement selection schemes shows that the present technique can identify multiple damages of the structure more accurately. The effect of the measurement number is also investigated.
\end{abstract}

Key words: damage detection, vibration method, sensor placement, uncertainty.

\section{INTRODUCTION}

Vibration-based damage detection methods have been developed for decades (Doebling et al. 1996; Jiang et al. 2006; Yan et al. 2010; Xu and Xia 2012; Xu and $\mathrm{Wu}$ 2007; $\mathrm{Xu}$ et al. 2011). Uncertainties in finite element (FE) models and measurements may result in false damage identification (Farrar and Doebling 1998; Xia et al. 2002). Moreover, the maximum number of measurement locations is limited due to technological and economical reasons. The measurement locations and the modes that are included in the analysis significantly influence the accuracy of the damage identification results (Sanayei and Saletnik 1996; Gunes et al. 1999; Xu and Wu 2009). Therefore, a proper measurement subset needs to be chosen carefully before field testing and damage identification analysis to ensure successful and efficient damage identification.
Several methods have been developed to determine the optimal measurement set for modal testing based on active vibration control theories. For example, Lim (1992) developed a method to select optimal actuator and sensor locations based on the degree of effectiveness/versatility of actuator-sensor pairs. Kammer (1991) presented a sensor placement method for modal identification and correlation according to the contribution of each candidate sensor location to the linear independence of the corresponding target modes. Breitfeld (1996) found that the optimal set of measurement points must preserve the orthogonality of the eigenvectors to avoid spatial aliasing. Meanwhile, Yi et al. (2011) proposed an optimal sensor placement by minimizing the maximum value of the off-diagonal elements of the modal assurance criterion matrix. Kammer (2005) conducted a comprehensive survey of sensor placement strategies for aerospace applications. 
At present, however, measurement selection for damage identification has not been sufficiently studied. Sanayei and Saletnik (1996) developed a best-in-worstout approach to select a subset of static force and strain measurements with the least sensitivity to measurement noise. The available measurement with the smallest effect on the parameter estimation is removed one by one until the output error becomes significant. Doebling et al. (1997) studied the mode selection strategy in locating damage and concluded that a mode selection based on maximum modal strain energy produced more accurate results than that based on minimum frequency. Cobb and Liebst (1997) proposed the optimal sensor placement for structural damage detection by maximizing system observability. A damage detection method of sensor location optimization, in which the collected information is used, has been presented by Shi et al. (2000). In their proposed method, the sensor locations are prioritized according to their contribution to a Fisher information matrix. Xia and Hao (2000) proposed a new concept of damage measurability that integrates damage sensitivity and noise sensitivity.

The current paper extends the study of Xia and Hao (2000) and proposes a new sensor placement indicator for damage detection. The new indicator is the ratio of two parameters. The first one is based on the work of Shi et al. (2000), namely, the contribution of measurement points to a Fisher information matrix, and the second one is the damage sensitivity to the measurement noise. The points that result in large indices are chosen as the measurement set. This selection technique is derived from the undamaged structure; thus, it is independent of the damage configuration. The proposed method can be applied to field modal testing and damage detection analysis. A laboratory-tested steel frame is studied to verify the effectiveness of the proposed method.

\section{FISHER INFORMATION MATRIX}

Let $\lambda_{i}$ and $\left\{\phi_{i}\right\}$ be the $i^{t h}$ eigenvalue and mode shape vector $(n p \times 1)$ at the corresponding $n p$ measured degrees of freedom (DOFs) of the intact structure, $\widetilde{\lambda}_{i}$ and $\left\{\widetilde{\phi}_{i}\right\}$ are the corresponding quantities of the damaged structure, respectively, $\{\alpha\}$ and $\{\tilde{\alpha}\}$ are structural element stiffness parameters of the initial and damaged FE models with ne elements. The measured vibration characteristics and the structural stiffness parameters can be expressed as a truncated Taylor series expansion given by:

$$
\left\{\frac{\tilde{\lambda}_{i}}{\tilde{\phi}_{i}}\right\}=\left\{\frac{\lambda_{i}}{\phi_{i}}\right\}+\left[S_{i}\right](\{\tilde{\alpha}\}-\{\alpha\})
$$

where $\left[S_{i}\right]$ is the corresponding sensitivity matrix with the following elements:

$$
\left[S_{i}\right]=\left[\begin{array}{l}
\frac{\partial \lambda_{i}}{\partial \alpha} \\
\frac{\partial \phi_{i}}{\partial \alpha}
\end{array}\right]
$$

The derivatives of the eigenvalue and mode shape with respect to the stiffness parameter can be calculated according to Nelson's method (1976).

Eqn 1 can be written as:

$$
\left\{e_{i}\right\}=\left[S_{i}\right]\{\Delta \alpha\},
$$

where $\left\{e_{i}\right\}$ is the modal data change vector containing the differences between the $i^{\text {th }}$ eigenvalue and mode shape of the structure before and after damage, and $\{\Delta \alpha\}$ is the element stiffness change which is solved by the least-squares method given by:

$$
\{\Delta \alpha\}=\left(\left[S_{i}\right]^{T}\left[S_{i}\right]\right)^{-1}\left[S_{i}\right]^{T}\left\{e_{i}\right\}
$$

Negative values in $\{\Delta \alpha\}$ denote location and severity of the structural damage. The detected damage is subject to error, because of the inevitable uncertainties in the measurement $\left\{e_{i}\right\}$, namely, frequencies and mode shapes.

The covariance matrix of the estimation errors should be minimized to accurately estimate the damage (or stiffness change) from Eqn 4. To achieve this, the Fisher information matrix $A$ given below should be minimized, according to the studies of Udwadia and Garba (1985):

$$
\left[A_{i}\right]=\left[S_{i}\right]^{T}\left[S_{i}\right]
$$

Eqn 5 illustrates that information is subsequently added to or subtracted from the Fisher information matrix as each DOF or sensor location is added or subtracted from the candidate set. Given that each DOF contribution is different, the norm of the information matrix when DOFs are removed from the candidate measurement set should be maintained. The points with small contributions to the Fisher information matrix are removed from the candidate measurement set, whereas those with large contributions are retained.

With an eigenvalue analysis, Kammer (1991) suggested that the diagonal terms of the following 
matrix $\left[E_{i}\right]$ could rank the importance of sensor location within the initial candidate set:

$$
\left[E_{i}\right]=\left[S_{i}\right]\left(\left[S_{i}\right]^{T}\left[S_{i}\right]\right)^{-1}\left[S_{i}\right]^{T}
$$

Hence, if a point contributes least to the diagonal terms of the matrix $\left[E_{i}\right]$, it is considered redundant and can thus be removed from the candidate measurement set. The remaining optimum subset is used to maximize the contribution to the Fisher information matrix. Measurements on these points give the most information for damage detection.

For a multiple-DOF system, the sensitivity matrices $\left[S_{i}\right]$ differ for different modes. Thus, each point contribution to the Fisher information matrix depends on the mode used. A summation of all available modes $\left[E_{i}\right]$ is used in this study. Vector $\left\{F_{i}\right\}$ is defined as the diagonal terms of the matrix:

$$
\left\{F_{i}\right\}=\operatorname{diag}\left(\left[E_{i}\right]\right)
$$

then the summation of $\mathrm{nm}$ modes results in a contribution vector given by:

$$
\{F\}=\sum_{i=1}^{n m}\left\{F_{i}\right\}
$$

\section{NOISE SENSITIVITY MATRIX}

In this study, individual candidate location and the noise influence from the measurements at the corresponding points are considered. In this regard, the noise sensitivity is defined as:

$$
\left[S_{i}^{n}\right]=\frac{\partial\{\Delta \alpha\}}{\partial\left\{X_{i}\right\}},
$$

where $\left\{X_{i}\right\}$ is a noise vector including the noise from the $i^{\text {th }}$ measured mode. The physical meaning of $\left[S_{i}^{n}\right]$ is the change in the identified $\{\Delta \alpha\}$ by a unit measurement noise. Measurement errors mainly come from procedures and equipment related to modal testing. The information about the errors from the field measurement is usually difficult to obtain; therefore numerical methods have been applied to estimate the uncertainties in the measured modal parameters. Katafygiotis and Yuen (2001), for example, estimated the modal parameters of structures using ambient data and the associated uncertainties using the Bayesian spectral density approach. The probabilistic distribution of the modal parameters could be well approximated as a Gaussian distribution centered at the optimal estimated parameters. Two numerical examples have been presented to show that the relative error of frequency is about 0.3\%. Doebling et al. (1997) estimated the relative uncertainties of the measured modal frequencies and mode shapes arising from random error sources by Monte Carlo analysis. Application to a real steel bridge showed that the relative error of frequency is about $0.1 \%$ to $0.7 \%$ and that of mode shape is about $2 \%$ to $40 \%$. Without losing generality, the relative error of mode shape is set to $10 \%$ in the present analysis.

For $\mathrm{nm}$ available modes, Eqn 3 is arranged as:

$$
\{e\}=[S]\{\Delta \alpha\},
$$

where $\{e\}$ and $[S]$ are combinations of $\left\{e_{i}\right\}$ and $\left[S_{i}\right]$ of $\mathrm{nm}$ modes, respectively. Taking derivatives of both sides of Eqn 10 with respect to a noise vector $\left\{X_{i}\right\}$, it has

$$
\frac{\partial\{e\}}{\partial\left\{X_{i}\right\}}=[S] \frac{\partial\{\Delta \alpha\}}{\partial\left\{X_{i}\right\}} .
$$

When only the measurement noise is considered and the error from the FE model is not included, the noise sensitivity is expressed as:

$$
\left[S_{i}^{n}\right]=\frac{\partial\{\Delta \alpha\}}{\partial\left\{X_{i}\right\}}=([S])^{+}\left(\frac{\partial\{e\}}{\partial\left\{X_{i}\right\}}\right) .
$$

For one mode, matrix $\left[S_{i}^{n}\right]$ has dimensions of $n e \times n p$. The $(j, k)$ term of matrix $\left[S_{i}^{n}\right]$ is the change in the $j^{\text {th }}$ element stiffness parameter when there is a unit noise in the measured data at the $k^{\text {th }}$ DOF. Given that damage can exist in any element, the absolute values of all the terms in the $k^{\text {th }}$ column are added to obtain the total noise sensitivity value of the $k^{\text {th }} \mathrm{DOF}$, which represents the influence of noise from the measured data at the $k^{\text {th }} \mathrm{DOF}$ on all element stiffness parameters. Consequently, it has

$$
\left\{S_{i}^{n}\right\}=\sum_{j=1}^{n e}\left|\left[S_{i}^{n}\right]_{j k}\right|
$$

For $\mathrm{nm}$ modes, the noise sensitivity vectors of all modes are added together as

$$
\left\{S^{n}\right\}=\sum_{i=1}^{n m}\left\{S_{i}^{n}\right\}
$$




\section{SENSOR PLACEMENT}

From the above definition, a large contribution vector value represents corresponding measurement points that contribute more to structural damage identification. On the other hand, a small noise sensitivity vector value indicates that the noise of corresponding measurement points has less influence on the identification results. In theory, points corresponding to the largest information contribution and smallest noise sensitivity should be used in the damage identification analysis. A large information contribution assures true damage identifiability, whereas small noise sensitivity makes the results more reliable. Both vectors are very important and should be integrated.

Therefore, one should select the measurement locations that give a large $\{F\}$ and small $\left\{S^{n}\right\}$ to achieve more accurate and reliable damage identification results. A sensor placement index is thus defined as the ratio of these two parameters given by:

$$
\{I\}=\frac{\{F\} / \max (\{F\})}{\left\{S^{n}\right\} / \max \left(\left\{S^{n}\right\}\right)} .
$$

Here the contribution vector and noise sensitivity vector are respectively normalized with respect to their corresponding maximum values. The points with larger sensor placement indices are then selected as the potential sensor locations.

Both $\{F\}$ and $\left\{S^{n}\right\}$ are calculated in the undamaged state. Thus, the present selection technique is independent of the damage configuration, making it feasible in performing damage detection analysis.

\section{EXPERIMENTAL STUDY: A PORTAL FRAME}

\subsection{Introduction to the Experiment}

A laboratory tested portal frame is employed here to demonstrate the presented measurement selection algorithm. The cross section of the beam was $40.50 \times$ $6.0 \mathrm{~mm}^{2}$, and the columns were $50.50 \times 6.0 \mathrm{~mm}^{2}$; the mass density was $7.67 \times 10^{3} \mathrm{~kg} / \mathrm{m}^{3}$ (Figure 1). The bottom parts of the columns were welded onto a thick plate (about $1.20 \mathrm{~m} \times 1.20 \mathrm{~m} \times 15.0 \mathrm{~mm}$ ), which was clamped on the floor to model the fixed boundary condition. Two damage scenarios were introduced by saw cuts at four different locations. The cut depths were about $20 \%$ of the section height for the first damage case and $30 \%$ for the second.

The intact configuration D0 and the damaged configurations (D1 and D2) were tested with an

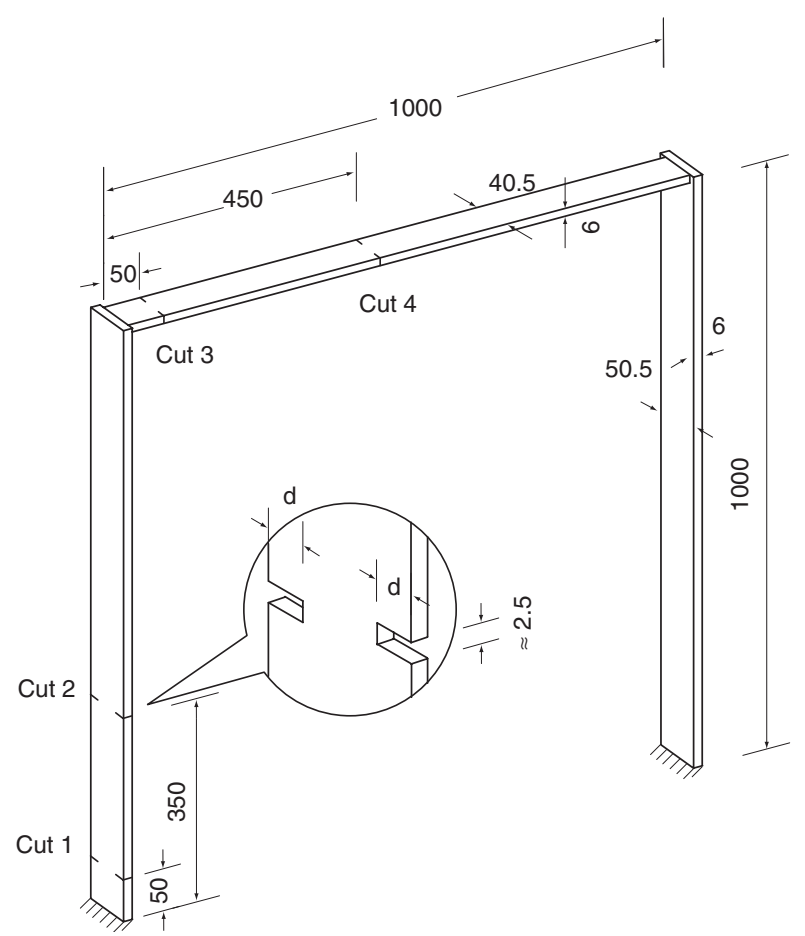

Figure 1. Configuration of the frame specimen (unit: $\mathrm{mm}$ )

instrumented hammer. Accelerations at 29 points (shown in Figure 2 except the fixed supports) were recorded in each configuration. The test was repeated four times to average the signals. The sampling frequency was $600 \mathrm{~Hz}$, and the cutoff frequency was $256 \mathrm{~Hz}$ with a resolution of $0.1465 \mathrm{~Hz}$. The in-plane vibration frequencies and mode shapes at 29 points were

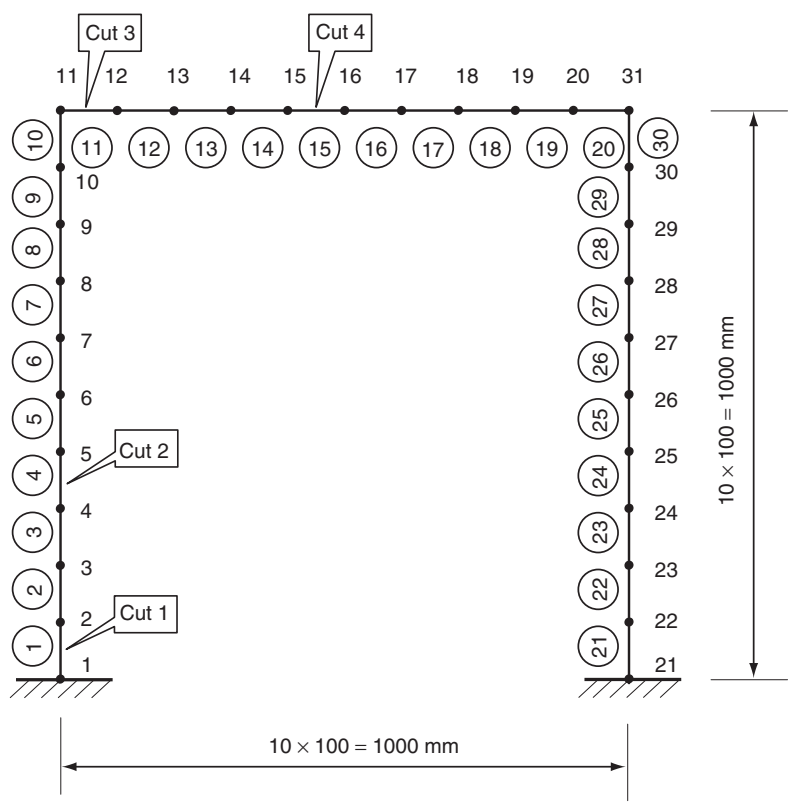

Figure 2. Finite element model of the frame 
extracted from the frequency response functions by the nonlinear least-square method with a commercial software ICATS (1998). Details of the experiment can be referred to Hao and Xia (2002).

In the experiment, the first 12 frequencies and mode shapes at all 29 points were used to detect four artificial damages. The frequencies of the frame in different states and the proportional changes are listed in Table 1. The average frequency change values are about $0.5 \%$ and $1.3 \%$ for the two damage scenarios, respectively. The mode shape changes are negligible and are not shown in this paper. The example was used to select a measurement subset to reduce the measurement points in the modal test.

\subsection{Sensor Placement Index}

The FE model of the frame had 30 Euler-Bernoulli beam elements (i.e., $n e=30$ ), as shown in Figure 2. The Young's modulus was $200 \mathrm{GPa}$. The element bending rigidity was considered as the element stiffness parameter. The sensitivity matrix was computed from the FE model. Then the contribution vectors of 12 modes $(n m=12)$ are obtained from Eqns 6 and 7. The contribution vector was derived from Eqn 8 and then listed in Table 2. The noise sensitivity matrix was estimated for each point according to Eqn 12 and summed up for one mode as Eqn 13. The noise sensitivity vector for the first 12 modes was calculated using Eqn 14 and then listed in Table 2. The sensor placement index was also computed. The results are listed in Table 2.

\section{Table 1. Experimental frequencies of the frame in different states $(\mathrm{Hz})$ and frequency change ratios (\%)}

\begin{tabular}{lcrr}
\hline & $\begin{array}{c}\text { Undamaged } \\
\text { state }\end{array}$ & \multicolumn{2}{c}{ Damaged states } \\
\hline Mode & D0 & D1 & \multicolumn{1}{c}{ D2 } \\
\hline 1 & 4.49 & $4.46(-0.67)$ & $4.41(-1.78)$ \\
2 & 17.41 & $17.31(-0.57)$ & $17.16(-1.44)$ \\
3 & 27.99 & $27.78(-0.75)$ & $27.46(-1.89)$ \\
4 & 30.89 & $30.86(-0.10)$ & $30.28(-1.97)$ \\
5 & 61.84 & $61.69(-0.24)$ & $61.43(-0.66)$ \\
6 & 74.41 & $73.72(-0.93)$ & $72.91(-2.02)$ \\
7 & 87.79 & $87.30(-0.56)$ & $86.75(-1.18)$ \\
8 & 132.99 & $132.41(-0.44)$ & $131.61(-1.04)$ \\
9 & 155.42 & $154.98(-0.28)$ & $154.08(-0.86)$ \\
10 & 165.67 & $165.10(-0.34)$ & $164.30(-0.83)$ \\
11 & 228.70 & $227.93(-0.34)$ & $227.20(-0.66)$ \\
12 & 255.30 & $254.10(-0.47)$ & $252.15(-1.23)$ \\
Average of changes $(\%)$ & -0.47 & -1.30 \\
\hline
\end{tabular}

( ) are the frequency change ratios (\%) between the damaged states and the undamaged state
Table 2. Sensor placement selection of the frame

\begin{tabular}{|c|c|c|c|}
\hline $\begin{array}{l}\text { Point } \\
\text { (1) }\end{array}$ & $\begin{array}{l}\text { Contribution } \\
\text { vector } \\
\{F\} / \max (\{F\}) \\
\text { (2) }\end{array}$ & $\begin{array}{c}\text { Noise } \\
\text { sensitivity } \\
\left\{S^{n}\right\} / \max \left(\left\{S^{n}\right\}\right) \\
\text { (3) }\end{array}$ & $\begin{array}{c}\text { Sensor } \\
\text { placement } \\
\text { index }\{/\} \\
(4)=(2) /(3)\end{array}$ \\
\hline 2 & 0.243 & 0.443 & 0.549 \\
\hline 3 & 0.276 & 0.972 & 0.284 \\
\hline 4 & 0.550 & 0.786 & 0.699 \\
\hline 5 & 0.671 & 0.878 & 0.764 \\
\hline 6 & 0.617 & 0.683 & 0.903 \\
\hline 7 & 0.405 & 0.848 & 0.478 \\
\hline 8 & 0.607 & 0.849 & 0.715 \\
\hline 9 & 0.436 & 0.810 & 0.538 \\
\hline 10 & 0.351 & 0.696 & 0.505 \\
\hline 11 & 0.346 & 0.070 & 4.924 \\
\hline 12 & 0.579 & 0.738 & 0.784 \\
\hline 13 & 0.609 & 0.939 & 0.649 \\
\hline 14 & 0.485 & 0.924 & 0.525 \\
\hline 15 & 0.425 & 1.000 & 0.425 \\
\hline 16 & 1.000 & 0.703 & 1.423 \\
\hline 17 & 0.433 & 0.994 & 0.436 \\
\hline 18 & 0.473 & 0.923 & 0.512 \\
\hline 19 & 0.617 & 0.935 & 0.660 \\
\hline 20 & 0.556 & 0.751 & 0.739 \\
\hline 22 & 0.253 & 0.446 & 0.567 \\
\hline 23 & 0.264 & 0.991 & 0.266 \\
\hline 24 & 0.538 & 0.802 & 0.670 \\
\hline 25 & 0.607 & 0.875 & 0.695 \\
\hline 26 & 0.559 & 0.687 & 0.814 \\
\hline 27 & 0.385 & 0.844 & 0.457 \\
\hline 28 & 0.584 & 0.850 & 0.688 \\
\hline 29 & 0.423 & 0.801 & 0.529 \\
\hline 30 & 0.358 & 0.697 & 0.514 \\
\hline 31 & 0.339 & 0.070 & 4.825 \\
\hline
\end{tabular}

\subsection{Damage Detection}

Four measurement subsets based on different sensor placement schemes were selected. The first set (Set 1) selected the points with the highest contribution values as used by Shi et al. (2000). The second one (Set 2) selected the points with the lowest noise sensitivity values. The third one (Set 3 ) was based on the proposed sensor placement index. The last one (Set 4) consisted of every other point in the FE model. The points corresponding to the four subsets are listed accordingly in Table 3. In each subset, the first 12

Table 3. Details of the measurement points

\begin{tabular}{lcc}
\hline Set & Points & Quantity \\
\hline 1 & $5,6,8,12,13,16,19,20,25,26,28$ & 11 \\
2 & $2,6,10,11,12,16,20,22,26,30,31$ & 11 \\
3 & $4,5,6,8,11,12,16,20,25,26,31$ & 11 \\
4 & $3,5,7,9,11,13,15,17,19,23,25,27,29,31$ & 14 \\
\hline
\end{tabular}


mode shapes measured at the corresponding points and the frequencies were used to detect damages with an optimal model updating approach (Hao and Xia 2002). The detection results for the two damage scenarios are compared in Figures 3 and 4, respectively. Here the ordinate $S R F$ denotes the stiffness reduction factor of each element and is defined as the ratio of element stiffness reduction to the initial element stiffness parameter in the undamaged state. This is expressed as:

$$
S R F_{i}=\frac{\Delta \alpha_{i}}{\alpha_{i}}
$$

where $i=1,2, \ldots, n e$. Lower and upper bounds were imposed during damage detection to ensure that the updated stiffness maintained valid physical meaning or the damaged stiffness parameter was neither larger than the undamaged one nor negative. This is expressed as:

$$
-1<S R F_{i} \leq 0,
$$

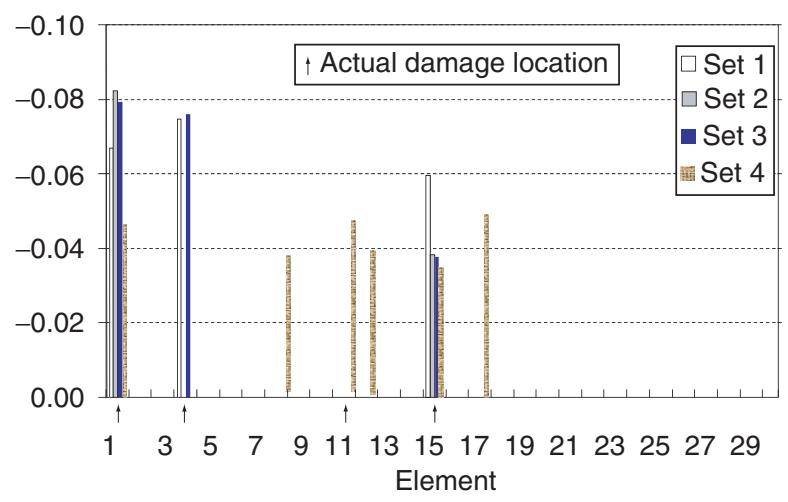

Figure 3. Elemental SRF of D1 with different measurement subsets

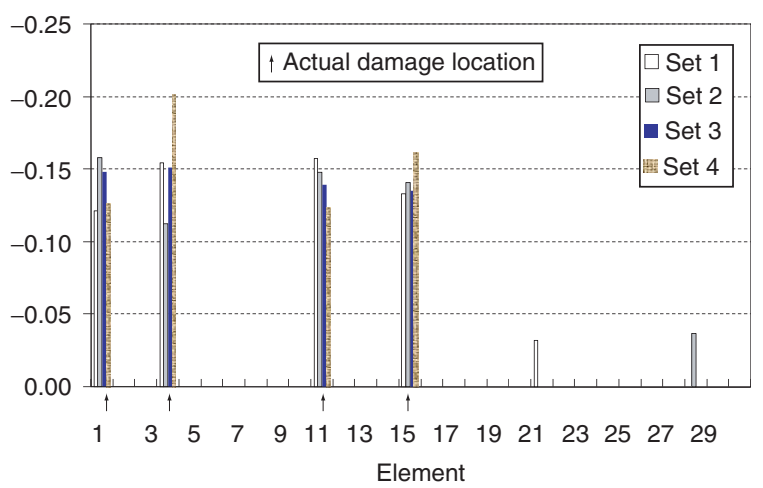

Figure 4. Elemental $S R F$ of D2 with different measurement subsets where $S R F$ represents the damage severity in a certain element. There is no damage in the element if its $S R F$ is equal to zero, whereas there is total loss of the element stiffness if its $S R F$ equals -1 .

Sets 1 to 3 have 11 points, which are less than the 14 points in Set 4. Later results will show that Set 3, however, gives more accurate damage identification results even with fewer points.

For the damage identification results using four different subsets, the negative falsity (true damaged element not detected) and positive falsity (undamaged element detected as damaged) will be evaluated. An element with an absolute $S R F$ value less than $3 \%$ is considered undamaged; otherwise, it is considered damaged. For example in the first damage state D1 (Figure 3), the damaged Element 4 is not detected but Elements 8, 12, and 17 are wrongly detected as damaged with the measurement Set 4 . The numbers of negative falsity and positive falsity using Set 4 are 1 and 3, respectively. Similarly the numbers of negative falsity and positive falsity using Set 1, Set 2, and Set 3 are 1, 0; 2 , 0 ; and 1, 0 , respectively. Therefore, using the measurement Set 1 or Set 3 results in least false identification.

Figure 4 demonstrates the $S R F$ corresponding to a more severe damage state D2 with the different subsets. Four damaged elements are detected correctly. There is no false detection with Set 3 or Set 4, but one element is wrongly detected with Set 1 or Set 2.

From the above observations, it can be concluded that, with the measurement points derived by the proposed technique, most damaged elements can be identified more accurately and reliably than the optimal sensor placement method by Shi et al. (2000) or by placing sensors at randomly selected measurement locations. In addition, all the four sensor placement schemes considered in the present study provide more accurate damage detection results for severe damage. This is because the measurement noise has a relative smaller effect on damage detection when the damage becomes severe, thus the damage identifiability is also improved.

\subsection{Effect of Sensor Numbers}

A total of 11 points were selected in the previous section for Sets 1 to 3 . This section investigates the reliability of damage detection when less measurement points, such as eight, are used. According to Table 2, eight points with the highest contribution vector values are chosen as Set 1, eight points with the lowest noise sensitivity vector values are chosen as Set 2, and eight points with the highest sensor placement indices are chosen as Set 3. Eight 
uniformly distributed points are chosen as Set 4 . Table 4 lists these points for each set.

Similar model updating procedures are then applied to detect the damage of the frame using different measurement subsets. The damage detection results for two damage scenarios are shown in Figures 5 and 6, respectively. Again, an element with an absolute $S R F$ value less than $3 \%$ is regarded as undamaged. Figure 5 shows that Set 1 falsely detects Element 7 as damaged and misses damage detection at Elements 11 and 15 . Set 2 falsely detects Element 27 as damaged and misses damage detection at Elements 4, 11 and 15. Set 3 has no false detection but misses damage detection at

Table 4. Measurement selection with fewer points

\begin{tabular}{lcc} 
Set & Points & Quantity \\
\hline 1 & $5,6,8,13,16,19,25,28$ & 8 \\
2 & $2,6,10,11,22,26,30,31$ & 8 \\
3 & $5,6,11,12,16,20,26,31$ & 8 \\
4 & $4,7,11,14,18,24,27,31$ & 8 \\
\hline
\end{tabular}

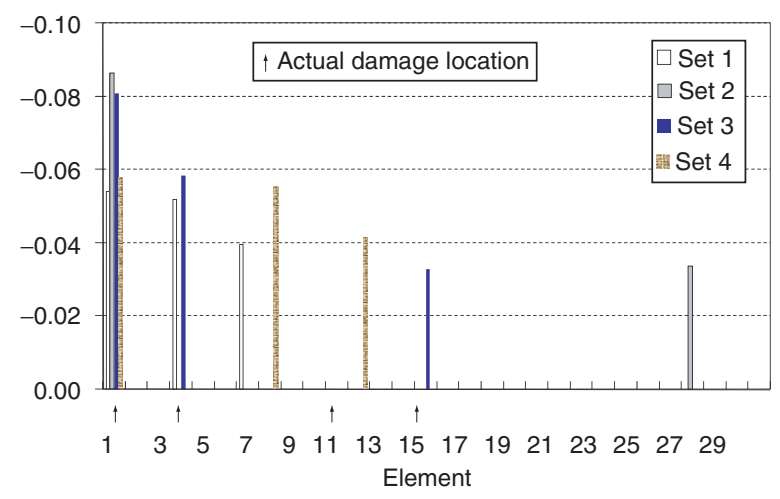

Figure 5. Elemental $S R F$ of D1 with different measurement subsets (eight measurement points)

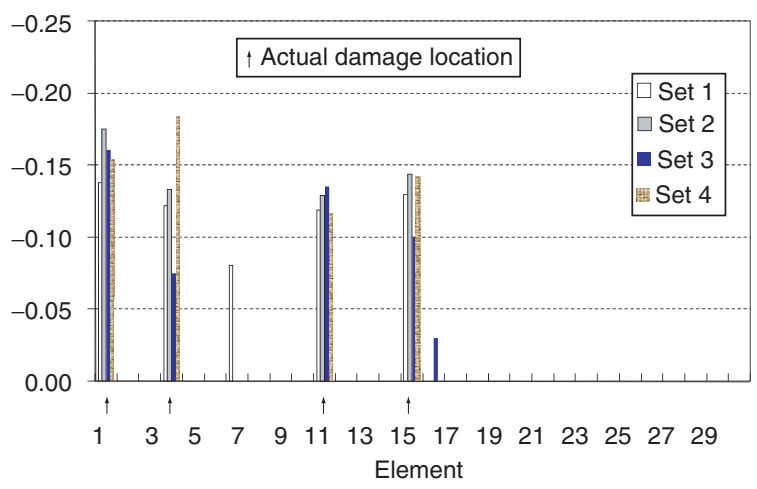

Figure 6. Elemental $S R F$ of D2 with different measurement subsets (eight measurement points)
Element 11 only. Set 4 incorrectly detects two elements (Nos. 8 and 12) as damaged and misses detecting the damages at Elements 4, 11, and 15. These results demonstrate that the present measurement selection technique renders the best performance in terms of damage detection.

In the case of severe damage D2, as shown in Figure 6, all four sets can detect damage successfully, although Sets 1 and 3 have a slight false detection. As explained before, the measurement noise has less influence on detecting severe damages.

Comparisons between Figures 3 and 5 and between Figures 4 and 6 show that using fewer points does not significantly affect the damage detection results. To demonstrate this further, all the measured 29 points were used for damage detection. The results for D1 and D2 are plotted in Figure 7. For damage state D1, using all measurement points results in several false detections and misses damage detection at Element 4. The overall performance of reduced points in damage detection is similar to that of using 14 points randomly, but worse than that using the present measurement selection technique. Again using all measurement points can successfully detect the severe damage D2.

At present, no attempt has yet to be made to further reduce the measurement points because of the difficulty in determining the least number of measurement points. Theoretically speaking, if the total number of known measurements is greater than the number of unknown parameters, the model updating process is a determined inverse problem, and the damage can be correctly detected regardless of the selected measurement points. Because of the measurement noise, including more points, however, may cause the inverse problem illconditioned and lead to incorrect damage detection results. The present technique aims to select the points with less negative effects on damage detection.

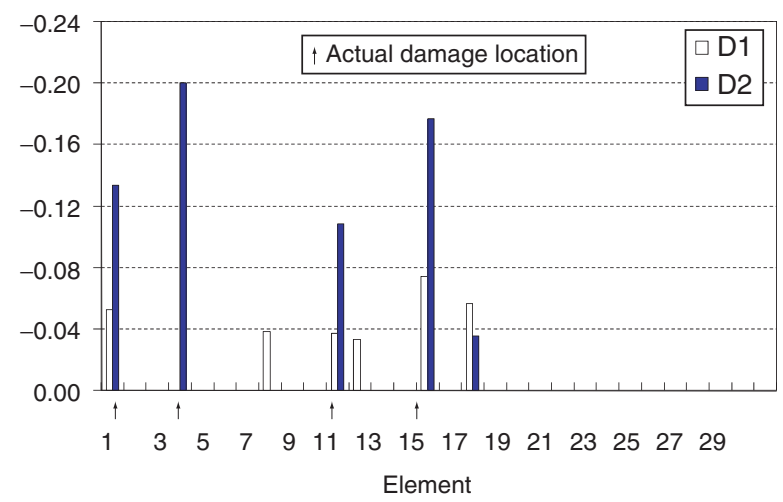

Figure 7. Elemental $S R F$ s of D1 and D2 with all 29 measurement points 


\section{CONCLUSIONS}

Although extensive research has been conducted over the past decades, the vibration-based structural damage detection methods are still not matured. Uncertainties in the structural model and measured vibration data possibly lead to unreliable damage detection. With respect to modal testing, measurement noise is inevitable, and the maximum number of measurement locations is limited. Although more measurement points provide more information, they also involve more noise at the same time. Therefore using more measurement points does not necessarily lead to better damage detections as compared to strategically selecting the measurement locations. The measurement locations greatly influence the accuracy of damage identification results.

In this work, a new method has been developed to derive a proper subset of measurement points for structural damage detection. The new method is based on the contribution of the measurement to damage detection and the noise sensitivity. The points with the largest contributions and smallest noise sensitivity should be selected in measurements for damage identification analysis. Applying the proposed procedure to a laboratory-tested frame demonstrated the improved accuracy of the damage identification results. The advantage of the present method is that it is derived from the undamaged structure, hence, the proposed method is independent of the damage configuration.

The present method has been verified through the simple portal frame only and it might need further study. In addition, the optimal sensor location and optimal number of sensors are worth of studying.

\section{ACKNOWLEDGMENTS}

The authors gratefully acknowledge the financial support provided by the National Natural Science Foundation of China (Project Nos. 51078235 and 50830203) and the Hong Kong Polytechnic University (Project No. G-YK74).

\section{REFERENCES}

Breitfeld, T. (1996). "A method for identification of a set of optimal measurement points for experimental modal analysis", Modal Analysis: The International Journal of Analytical and Experimental Modal Analysis, Vol. 11, No. 1, pp. 1-9.

Cobb, R.G. and Liebst, B.S. (1997). "Sensor placement and structural damage identification from minimal sensor information", AIAA Journal, Vol. 35, No. 2, pp. 369-374.

Doebling, S.W., Farrar, C.R., Prime, M.B. and Shevitz, D.W. (1996). Damage Identification and Health Monitoring of Structural and Mechanical Systems From Changes in Their Vibration Characteristics: A Literature Review, Los Alamos National Laboratory Report LA-13070-MS, Los Alamos, USA.
Doebling, S.W., Hemez, F.M., Peterson, L.D. and Farhat, C. (1997). "Improved damage location accuracy using strain energy-based mode selection criteria", AIAA Journal, Vol. 35, No. 4, pp. 693-699.

Doebling, S.W., Farrar, C.R. and Goodman, R.S. (1997). "Effects of measurement statistics on the detection of damage in the Alamosa Canyon Bridge", Proceedings of the $15^{\text {th }}$ International Modal Analysis Conference, Orlando, FL, USA, February, pp. 919-929.

Farrar, C.R. and Doebling, S.W. (1998). "A comparison study of modal parameter confidence intervals computed using the monte carlo and bootstrap techniques", Proceedings of $16^{\text {th }}$ International Modal Analysis Conference, Santa Barbara, CA, February, pp. 936-944.

Gunes, B., Arya, B., Wadia-Fascetti, S. and Sanayei, M. (1999). "Practical issues in the application of structural identification", Computational Mechanics and Structural Engineering: Recent Developments, Elsevier Scientific Ltd., pp. 193-206.

Hao, H. and Xia, Y. (2002). "Vibration-based damage detection of structures by genetic algorithm", Journal of Computing in Civil Engineering, ASCE, Vol. 16, No. 3, pp. 222-229.

Jiang, S.F., Zhang, C.M. and Koh, C.G. (2006). "Structural damage detection by integrating data fusion and probabilistic neural network", Advances in Structural Engineering, Vol. 9, No. 4, pp. 445-458.

Kammer, D.C. (1991). "Sensor placement for on-orbit modal identification and correlation of large space structures", Journal of Guidance, Control, and Dynamics, Vol. 14, No. 2, pp. 251-259.

Kammer, D.C. (2005). "Sensor set expansion for modal vibration testing”, Mechanical System and Signal Processing, Vol. 19, No. 4, pp. 700-713.

Katafygiotis, L.S. and Yuen, K.V. (2001). "Bayesian spectral density approach for modal updating using ambient data", Earthquake Engineering and Structural Dynamics, Vol. 30, No. 8, pp. 1103-1123.

Lim, K.B. (1992). "Method for optimal actuator and sensor placement for large flexible structures", Journal of Guidance, Control, and Dynamics, Vol. 15, No. 1, pp. 49-57.

MODENT (1998). User's Guide, ICATS, Imperial College Analysis, Testing and Software, London, UK.

Nelson, R.B. (1976). "Simplified calculation of eigenvector derivatives", AIAA Journal, Vol. 14, No. 9, pp. 1201-1205.

Sanayei, M. and Saletnik, M.J. (1996). "Parameter estimation of structures from static strain measurements, Part II: Error sensitivity analysis", Journal of Structural Engineering, ASCE, Vol. 122, No. 5, pp. 563-572.

Shi, Z.Y., Law, S.S. and Zhang, L.M. (2000). "Optimum sensor placement for structural damage detection", Journal of Engineering Mechanics, ASCE, Vol. 126, No. 11, pp. 1173-1179.

Udwadia, F.E. and Garba, J.A. (1985). “Optimal sensor locations for structural identification", Proceedings of the Workshop on Identification and Control of Flexible Space Structures, San Diego, California, USA, April, pp. 247-261. 
Xia, Y. and Hao, H. (2000). "Measurement selection for vibrationbased structural damage identification", Journal of Sound and Vibration, Vol. 236, No. 1, pp. 89-104.

Xia, Y., Hao, H., Brownjohn, J.M.W. and Xia, P.Q. (2002). "Damage Identification of structures with uncertain frequency and mode shape data", Earthquake Engineering and Structural Dynamics, Vol. 31, No. 5, pp. 1053-1066.

Xu, Y.L. and Xia, Y. (2011). Structural Health Monitoring of LongSpan Suspension Bridges, Spon Press, London, UK.

$\mathrm{Xu}, \mathrm{Z} . \mathrm{D}$. and Wu, Z.S. (2007). "Energy damage detection strategy based on acceleration responses for long-span bridge structures", Engineering Structures, Vol. 29, No. 4, pp. 609-617.

$\mathrm{Xu}, \mathrm{Z} . \mathrm{D}$. and Wu, Z.S. (2009). "Sensitivity analysis of accelerationbased energy damage detection strategy to load excitations and sensor placement", Journal of Intelligent Material Systems and Structures, Vol. 20, No. 4, pp. 413-423.

Xu, Z.D., Liu, M., Wu, Z.S. and Zeng, X. (2011). "Energy damage detection strategy based on strain responses for long-span bridge structures." Journal of Bridge Engineering, ASCE, Vol. 16, No. 5, pp. 644-652.

Yan, W.J., Huang. T.L. and Ren, W.X. (2010). "Damage detection method based on element modal strain energy sensitivity", Advances in Structural Engineering, Vol. 13, No. 6, pp. 1075-1088.

Yi, T.H., Li, H.N. and Gu, M. (2011). "A new method for optimal selection of sensor location on a high-rise building using simplified finite element model", Structural Engineering and Mechanics, Vol. 37, No. 6, pp. 671-684. 
